\title{
Exploratory Research to Identify the Characteristics of Cyber Victims on Social Media in New Zealand
}

\author{
Varun Dhond ${ }^{1}$, Shahper Richter ${ }^{1}$, Brad McKenna ${ }^{2}$ \\ ${ }^{1}$ Auckland University of Technology, Auckland, New Zealand \\ \{varun.dhond, shahper.richter\} eaut.ac.nz \\ ${ }^{2}$ University of East Anglia, Norwich, United Kingdom \\ b.mckenna@uea.ac.uk
}

\begin{abstract}
Cyberbullying is omnipresent among all sections of society who have access to the internet. Vast research has been carried out on this topic around the world however there has not been enough research that is New Zealand based. The objective of this research is to identify the characteristics of cyber victims on social media in New Zealand. We scrutinize the prevalence of cyberbullying in New Zealand among university students based on age, gender and personality. The survey was designed stating the hypotheses developed as a result of the literature review. We gathered the data of sample size $n=158$. We conclude that students with openness to experience are more likely to be cyberbullied compared to the other personalities. Whereas, we found no correlation of age and gender with the cyber bullying on a university level. The results from this study can have a positive application in counter cyberbullying programs in New Zealand. This study will a give an impetus for further analytical research in the field of cyber bullying in New Zealand.
\end{abstract}

Keywords: Social Media. Cyberbullying. Personality. Cyber Victims. New Zealand.

\section{Introduction}

The term "cyberbullying" is a combination of the cyber world and the traditional form of bullying. In the last decade, social media has been a major factor for social involvement not only among young adults but also among various other age groups [1]. When compared to the different age groups of the society bullying is more prevalent among the youth population [2]. Social media open a platform of opportunities for everyone, but they have flaws, the consequences of which have been stronger negative feelings and higher rates of depression which may result in the worst possible outcomes like suicide [3].

We define cyberbullying as the intentional or unintended harm imposed by a person to demean the social value of another through an electronic medium. In our research, when we say youth we are considering people between the ages of 18 and 30 years who are studying in universities in New Zealand. As indicated in US national data, approximately $15 \%$ of youth have been victims of cyberbullying [4]. As relevant to our research, from previous New Zealand based statistics, $2.6 \%$ among 826 participants were bullied in the span of six months in New Zealand where more males were victimized than females on social networking sites (SNS) [5].

Despite much research being carried out in the world, few studies have focused on New Zealand. The reason for that can be because it's a small country, lack of funding, or small-scale implementation of government policies. There has been studies [6],[7], which had targeted Australian adolescents, yet not many researchers has approached New Zealand's social media circuit. Hence, we attempt to identify the likely victims of cyberbullying in New Zealand based on their age, gender and personality. The aim of the research is to design a conceptual model based on factors to get the permutations and combinations of the persons most likely to be cyberbullied. This research can also be considered as a pilot as the data samples collected are 158. As the nature of this research is exploratory it is important because it's a preliminary stepping stone in New Zealand's social media and bullying co-relation. The following is the research question: What type of student (age, gender, personality) is most likely to be bullied 
on social media?

The next section is a literature review, which will provide us the perspective to design a hypothesis and carry out the research. Following that we present our methodology, findings and results, and finally we discuss and conclude the paper.

\section{$2 \quad$ Literature Review}

This section begins with assessing the core concepts of cyberbullying and how prevalent it is in society. We will investigate the concepts of cyberbullying, its victims, the causes, and the efforts so far to prevent it.

\subsection{Cyberbullying}

Both bullying and victimization are rampant in the society and have adverse effects on both the victim and the bully [8]. The concept of cyberbullying is defined by Patchin and Hinduja as "wilful and repeated harm imposed through the medium of electronic text" [9]. In the literature, we found nine different types of cyberbullying [1012] flooding, masquerade, flaming/bashing, trolling, harassment, cyberstalking of cyber threats, denigration, outing, and exclusion. Flooding comprises of a monopoly by the bully to avoid the victim posting the contents on social media [11]. Masquerade is an act of the bully to $\log$ in to social media to use the credentials of the victim to post contents online and chat with other people, causing a threat to others to hamper the reputation of the victim [13]. Flaming or Bashing involves two users intensely involving in a heated argument and attacks on a personal level in public or in private.

This form of cyberbullying is usually short-lived [13]. Trolling implies posting an intentional disagreement with the purpose of provocation to an individual or group of people for engaging in an argument. It is not necessary that the disagreement is an actual opinion of the bully [10]. Harassment is the quintessential form of bullying which is a clichéd bully-victim relationship. This form of bullying involves sending offensive messages to the victim which can be prolonged depending on the actions taken over the period of time [13]. Cyberstalking and Cyber threats may involve sending intimidating, threatening or very abusive messages to the victim with an intention of threat or extortion [13]. Denigration is the spreading of untrue or foul rumours about someone in the public domain online. It also involves gossiping about the victims on the public domain and derogating their image online [13]. Outing is identical to denigration, requiring the bully to have a personal relationship with the victim. In this form of bullying, the bully posts private, personal and embarrassing information about the victim online [13]. Exclusion is ignorance towards the victim in public domains or chat rooms, isolating them leading to psychological distress [14].

From all the types mentioned above, cyberbullying can be caused by ignorance as well as on purpose. While Masquerade, Trolling, Harassment, Cyberstalking, and Outing are intentional forms of cyberbullying, Flaming be an unintentional cyberbullying as someone in a bad state of mind can cause it by unknowingly demeaning someone's social value. Denigration can be both intentional and unintentional as the person starting the hoax can do it intentionally, but the others can pass on the information due to ignorance. Historically, the traditional form of bullying was considered to be an acceptable part of a childhood [15]. Research by [16] theorizes that the repercussions of cyber victimization can be even more hazardous compared to face-to-face bullying. There can be a social ineffectiveness among victims who also face greater interpersonal anxiety $[17,18]$. Yet it is difficult to jump to the conclusion that these are the antecedents or consequences of cyberbullying [19, 20]. Compared to traditional bullying, cyberbullying can reach a wider spectrum of victims. For example, the traditional form of bullying can be among a small group of people or a school at maximum and not much evidence is kept circulating around, but the victims of cyberbullying can be the humiliated on a social platform in front of their friends, friends of friends, their family and people can share this act among the people whose numbers are difficult to estimate.

As mentioned in one article [21], the statistics are astounding and are New Zealand based. The Otago-based group named 'Sticks n stones' has surveyed 750 people of which $87 \%$ had been victims of cyberbullying. The most frequent victims were 
teenagers aged 18 and 19, of which $46 \%$ have faced cyberbullying. According to NZ attitude and value studies, $27 \%$ of those aged 20 to 24 years had been victimized whereas those from age 25 to 29 years have faced cyberbullying in some form or the other.

\subsection{Cyber Victimization}

Peer victimization is not a new concept. Several studies have found multiple peer victimizations such as physical attack, verbal harassment, social exclusion, spreading rumours and cyberbullying [22-24]. Cyberbullying/victimization is the newest of all and is our area of research. A study by [25], found a reciprocal relationship between bullying and victimization. The following study also stated that cyber bullies are also cyber victims at some point in their lives [26]. Different victims are also likely to handle the situation in a different manner: one is likely to take the scenario sportingly by overlooking the whole instance, while some might get offended but will not react to the scenario to maintain their dignity and some could also lose their psychological stability leading to actions like suicide, revenge, threats and self-destructive violence.

In research by [14], we have come across another perspective of children carrying the scars of cyberbullying from their childhood into their adulthood. It urges researchers to gain a better understanding of the antecedents and consequences of the bullying behaviour so that someone can come up with an antidote to the poison named cyberbullying. It was also an interesting finding, because the research was carried out over an online survey and most of the participants were teens and the majority of those were females. The findings clearly state that there is an occurrence of bullying among youth. Multiple occurrences of cyberbullying are prevalent among the youth from the study which includes being disrespectful, social avoidance, threatening etc. Studies have theorized the phenomena of cyberbullying being related to the behaviour of the victims [26-28]. One such study by [29], proposes the aggressive behaviours do not decrease over the course of time but instead, just take the shape of the mould it is currently accessible to. This idea leads us to the conclusion that people with more aggression who were used to traditionally victimising people, when they got access to the internet world are more likely to repeat the actions.

There is a rather interesting study by [30] which labels the characteristics of the person as a dark triad which can lead to them to be a cyber-bullying antagonist. This dark triad comprises Machiavellianism and narcissism. This is an interesting study because of how differently these triads lead to the same destination eventually. People with a Machiavellian triad possess manipulation as their basic characteristic [31]. This leads to cyber-aggression leading to cyber-victimization of one naïve enough to get trapped into the manipulative talks of a person with a Machiavellian personality. The next in the line is narcissism. The person possessing a narcissistic personality has a sense of eminence over others, which makes them a self-proclaimed authority to dominate or victimise others in social and cyber-space [32]. Related work has been carried out with this personality with reference to cyberbullying in the past which comprised anti-social behaviour on Facebook [33], as well as cyberbullying among youth [34].

Hence this research can give us more valuable and interesting insights when factors such as age and gender are taken together with personality to determine likely cyber victims.

\subsection{Causes of Cyberbullying}

Compared to the generation around two decades ago, because of the internet, youth today have an edge to be open to new experiences and satisfy themselves socially without socialising in person. Cyberbullying is the repercussion of this edge. In a research by [5], analysis of different motives like jealousy, bigotry, fear, anger, righteousness and revenge have been mentioned and this is just the tip of the iceberg. There can be so many other reasons for a person to commit cyberbullying. The abovementioned reasons can lead a person of specific age, gender or personality to become the victim of a cyberbully. Regardless of the vast variety in the frequency of cyberbullying, at reasonable and observational correlation is genuinely reliable [35]. Also, cyber bullies were found to show comparable patterns of psychological 
similarity as conventional harassers [36]. Hence, what can be the differentiation between traditional bullies and cyber bullies apart from the phone in the hand and internet access? The primary feature recognised is the obscurity that the internet gives, the social idea of the animosity, propelled internet knowledge, high recurrence of web utilisation, and its 24-hour reach [37, 38].

To continue with the argument, bullying others through electronic means furnishes the culprit with the likelihood of remaining unknown, which may build their power differential over the cyber-victim and in addition diminish the view of conceivable countering [39]. The social animosity of cyberbullying is additionally reflected in discoveries demonstrating that it frequently happens through SNS and is more regular among young girls [40].

\subsection{Past Efforts to Prevent Cyberbullying}

A study by [41], has a peculiar algorithm developed by analysing the theories by taking into consideration potential reasons for cyberbullying and a pathway for overcoming those. In their paper, two different theories have been proposed: The Neutralization theory and the Deterrence theory. The aim of the Neutralization theory is to figure out why people are more prone to cyberbullying. The Deterrence theory is an antidote to avoid cyberbullying as stated by the Neutralization theory. According to [41], the 3dimensionality of the Neutralization theory also signifies how the culprit defends his anamorphic actions. The basis of Deterrence lies in the two building factors, certainty and severity. In this scenario, certainty is termed as the risk of getting caught while in the act of cyberbullying, whereas severity states the sets of penalties to be imposed on committing the specific cyberbullying crime. Their paper has stated that the Neutralization theory has been effective in neutralizing cyberbullying among youth. In a similar context, it has been understood that there is a need to consider other risk variables like the ones at a family level.

In future, if efforts made to monitor the usage of substances, especially alcohol, amongst youth, come up with certain prevention programmes to make people aware of the harmful effects, this might help to show positive results. In the past, the application of data mining concepts and artificial intelligence have been applied to curtail cyberbullying [42]. This has been a recent finding to slow down cyberbullying by enforcing a framework to detect inappropriate content through an SVM linear classifier. Natural Language Processing (NLP) models such as Bag of Words (BoW), Latent Dirichlet Allocation (LDA) and Latent Semantic Analysis (LSA) have proven effective detecting cyberbullying [43]. This has been implemented on Twitter, where the classifier scrutinizes the data to expand the list of predefined words and organize them as per weights and priority to identify the bully features.

In the section above, we have covered the core concepts around cyber victimization. This allows us to gather a perspective and design a hypothesis in the next section.

\section{Hypothesis Development}

Based on the research questions discussed in the introduction, hypotheses were developed for this research.

\subsection{Age}

Age plays an important part in defining the person being cyber victimised. We must draw a hypothesis to propose a theory on which there can be a definite result obtained. "Age is just a number", but is it applicable to cyber victimization? If an individual uses the internet, they may be a victim irrespective of their age [44]. Although age is not a barrier to research for cyberbullying, many researchers have dedicated their time and effort to figure out cyberbullying among youth and its preventive measures. Researchers like [45], have some deep insights about cyber bully victimization among youth. Some studies claim to have disapproved the relatability of age with cyber victimization [46-49], whereas, on the contrary, there are also studies which validate the existence of cyber victimization existing among the youth, especially students [14, 50-53]. We would like to draw a hypothesis stating the vital role age can play for a student to be cyber victimised. 
H1: The age of the student on social media has a direct impact on them being cyber victimised.

Table 1 Hypothesis for Age

\begin{tabular}{ll}
\hline Hypothesis & Null Hypothesis \\
\hline $\begin{array}{l}\text { H1a: The younger the student the more is } \\
\text { the likelihood of cyber victimization. }\end{array}$ & $\begin{array}{l}\text { The age of the student is irrelevant for } \\
\text { likelihood to be cyber victimized. }\end{array}$ \\
\hline $\begin{array}{l}\text { H1b: The older the student the more is the } \\
\text { likelihood of cyber victimization. }\end{array}$ & $\begin{array}{l}\text { The age of the student is irrelevant for } \\
\text { likelihood to be cyber victimized. }\end{array}$ \\
\hline
\end{tabular}

\subsection{Gender}

Gender can also be a valuable variable when it comes to classifying the vulnerability of the population to be studied. A study published by [54] explicitly mentions that gender is an important factor to research for differentiating between cyber and traditional bullying. We can also draw a hypothesis that because females are difficult to be bullied in the public space, their chances of being cyberbullied are higher. By uncovering the literature on traditional bullying methods, it has been discovered that boys are more convoluted in both bullying as well as victimization [55-57]. Other studies have demonstrated females are at a higher risk of cyberbullying because of a lack of receptiveness to traditional bullying compared to the electronic media because females are more affected by bullying psychologically [58].

Based on information from the literature on cyber victimization and the research questions we designed, we can draw the following gender-based hypotheses:

H2: The gender of the student on social media has a direct impact on their being cyber victimised.

Table 2 Hypothesis for Gender

\begin{tabular}{ll}
\hline Hypothesis & Null Hypothesis \\
\hline $\begin{array}{l}\text { H2a: Females are more likely to be } \\
\text { cyber victimized. }\end{array}$ & $\begin{array}{l}\text { The gender of the student is irrelevant for } \\
\text { likelihood to be cyber victimized. }\end{array}$ \\
\hline $\begin{array}{l}\text { H2b: Males are more likely to be } \\
\text { cyber victimized. }\end{array}$ & $\begin{array}{l}\text { The gender of the student is irrelevant for } \\
\text { likelihood to be cyber victimized. }\end{array}$ \\
\hline
\end{tabular}

\subsection{Personality}

Amongst all the popular theories on personality, the big five factors of [59] are relevant as well as applicable to our research. McCrae and Costa Jr (1997), theorised there are five major personalities of human behaviour: openness, conscientiousness, extraversion, agreeableness and neuroticism.

People with openness to experience generally are open minded people [59]. They welcome new ideas and prefer moving out of their comfort zone and like experiencing new things. These people are also likely to be cyber victims because they tend to be vulnerable when alone while moving out of their comfort zone. People with conscientiousness are workaholics [59]. They possess the virtue of dutifulness and self-discipline. They are generally well- organized and are focused on achieving their goals. People with this personality are less likely to be a cyber-victim or a bully as they are cautious of their environment [59]. People with an extroverted personality are highly social people. They are friendly, attention seeking, enthusiastic and talkative. These kinds of people are most likely to be cyber victimised because of their outgoing nature [59]. The basic equation may suggest that the more the person is in contact with a social group, the more likely they are to be bullied. A study conducted by [60] also says that people with extroversion as their triad have more Facebook friends. The next personality is agreeableness. People with this personality are the compassionate ones. They have a happy-to-help attitude, are courteous, empathetic and unselfish. These people are least prone to cyber-victimisation because of their good behaviour socially. But on the contrary, researchers have also shown that people with openness are more likely to display personal information on social media [61]. The last personality is Neuroticism. The people with this personality are soft targets for cyber bullies because 
of their vulnerability of being emotionally unstable. They tend to react to very small things which can lead to feuds on social media. They can also be upset easily, and trivial issues can make them angry.

H3: The personality of the student has a direct impact on their being cyber victimised.

Table 3 Hypothesis for Personality

\begin{tabular}{|c|c|}
\hline Hypothesis & Null Hypothesis \\
\hline $\begin{array}{l}\text { H3a: Extroverts are more likely students to } \\
\text { be cyber victimized. }\end{array}$ & $\begin{array}{l}\text { The personality of a student is } \\
\text { irrelevant for likelihood to be cyber } \\
\text { victimized. }\end{array}$ \\
\hline $\begin{array}{l}\text { H3b: Neurotics are more likely students to } \\
\text { be cyber victimized. }\end{array}$ & $\begin{array}{l}\text { The personality of a student is } \\
\text { irrelevant for likelihood to be cyber } \\
\text { victimized. }\end{array}$ \\
\hline $\begin{array}{l}\text { H3c: Students with openness to experience } \\
\text { are more likely to be cyber victimized. }\end{array}$ & $\begin{array}{l}\text { The personality of a student is } \\
\text { irrelevant for likelihood to be cyber } \\
\text { victimized. }\end{array}$ \\
\hline $\begin{array}{l}\text { H3d: Students with agreeable personalities } \\
\text { are more likely to be cyber victimised. }\end{array}$ & $\begin{array}{l}\text { The personality of a student is } \\
\text { irrelevant for likelihood to be cyber } \\
\text { victimized. }\end{array}$ \\
\hline $\begin{array}{l}\text { H3e: Students with conscientious } \\
\text { personalities are more likely to be cyber } \\
\text { victimised. }\end{array}$ & $\begin{array}{l}\text { The personality of a student is } \\
\text { irrelevant for likelihood to be cyber } \\
\text { victimized. }\end{array}$ \\
\hline
\end{tabular}

In this section we have designed the hypotheses on which we will base a questionnaire to gather data for analysis. The next section will give us an insight into the step-by-step process of building the questionnaire and executing the online survey.

\section{$4 \quad$ Methodology}

Research in the area of cyber victimization is abundant in most technologically advanced countries [62]. Hence, using New Zealand based data, we will carry out our research using exploratory data analysis. The underlying objective of the research is "identifying the characteristics of cyber victims on social media in New Zealand". The three variables we will be discussing for identifying the characteristics of the cyber victims are age, gender and personality.

The main motive behind building this questionnaire is to narrow down the larger context to the most convenient form. The quality of the data obtained heavily relies on the questions in the survey. The two basic rules to designing a questionnaire are relevancy and accuracy [63]. In this context, relevancy means the researchers' understanding of the questions, whereas, accuracy can be defined as the layout of the questionnaire [63]. The questionnaire we will use for the survey is designed with these principles in mind.

For our research and constructing the questionnaire, we are setting the age limit at between 18 and 30 years. Hence to quantify the variable, we will be focusing on asking the age in our questionnaire as a choice between the ranges 18-21 years, 22-25 years, 26-30 years, and 30 years and above. This will give us an idea if the students are getting cyber victimized, the ideal age when the impact of cyber victimization can be maximum. We will also focus on the age-related activities on social media and the actions taken when cyber victimized. In our analysis, we are trying to ascertain whether gender plays a role in cyber victimization. To this end, we will explore whether a gender is more likely to be cyber victimised on social media, and if so, while doing a certain activity on that SNS. By the end of the analysis in this section, we will arrive at a conclusion of cyber victimization with reference to gender on social media and the actions preferred by certain gender when cyber victimized.

Recently efforts have been put into the study to figure out personalities of individuals. This has been an influential factor while determining both the cyber bully and cyber victim [64]. This factor could turn out to be the most interesting part of the study. The research carried out by [59] regarding the five traits from the five-factor 
model has always been linked to studies of social networking technologies [65]. The five- factor model determines the different personality types of individuals. These personality traits are explained in our hypotheses and will be used now to construct the final segment of our questionnaire. To determine the personalities of the students participating in the survey, we used the big five 15 item scales. These questions were successfully conceptualised and implemented in research done by [65]. The survey was also validated by extensive use of the German Socio-Economic Panel Survey [66]. Each of the five personality traits is assessed by three items. These items are merged to an average score of the respective big five dimensions. Thus, this survey will allow us to get a personality score. We will be using principal component analysis (PCA) in the tool $\mathrm{R}$ to derive the personality scores and correlate factors determining cyber victimization.

The survey was distributed by through social media, mobile applications and email. The posts and the email comprised of a hyperlink to the online survey which was created using the survey generating tool Qualtrics.

\section{$5 \quad$ Findings and Results}

In this section, we analyse the data we have collected through the questionnaire and explain cyber victimization in statistics. Initially, we will perform some descriptive statistics on the raw data. Later, we will perform the principal component analysis to determine the personality of each individual respondent. Based on this information, we can later perform the analysis on age and gender to analyse the responses on cyber victimization.

\subsection{Descriptive Statistics}

After completing the online survey, 211 responses were returned. The final number of complete responses used for data analysis was 158 . Almost half of respondent's ranges were between 22 and 25 years. The rest of the respondents are almost evenly distributed in the other age groups. The gender variable was also evenly distributed among males and females with $56.96 \%$ and $43.04 \%$ respectively. A noteworthy fact from the table is that $96.84 \%$ of university students use social media which made them ideal candidates to answer the cyberbullying questions.

Facebook is the most preferred social media site among the university students with $69.93 \%$ users. The next most popular is YouTube with $41.18 \%$ users, followed by Instagram, Snapchat and Twitter. The time spent on social media is somewhat even as the numbers lie close to each other, still, $36.60 \%$ students spend around 2 to 4 hours a week on social media. Watching others' activity is the most preferred activity for social media users, where $68.63 \%$ users prefer doing it. As we discussed in the literature review, to see how the youth in New Zealand prefer using social media, this analysis can be the answer to a certain extent. Chatting with others is also one of the preferred activities on social media, preferred by $44.44 \%$. The other activities $(9.15 \%)$ include watching videos, browsing through news feeds on social media, researching and browsing for memes.

When asked about being cyberbullied, $73.86 \%$ of the students had never been cyberbullied. Only $13.73 \%$ of the students were affirmative of being bullied whereas $12.42 \%$ were not sure of being cyberbullied. When looking into our findings, most cyberbullying happens on Facebook with $22.82 \%$. The rest of the social media sites are below $10 \%$.

\subsection{Age}

The rate of cyberbullying is low in New Zealand among university students. Students inall age groups were least bullied or they were unsure, but it is apparent that most of them were not bullied based on their age. 113 out of 153 respondents were not bullied, and $83.33 \%$ in the age group of 31 years and above were least bullied. Looking at the bullying rate in New Zealand, the most likely to be bullied are from ages 18 to 21 years and 26 to 30 years with $22.22 \%$ and $23.08 \%$ respectively. One of the noteworthy points is, the number of students saying maybe is like that of the number of students bullied. This gives a vague idea that they either don't want to reveal whether they are 
bullied, or they might be unclear about the fact of having been bullied on SNS. The $(\mathrm{p}=)$ value for this hypothesis 0.32 which is $(p>0.05)$. Hence, we can accept the null hypothesis for $\mathrm{H} 1$ i.e. the age of the students is irrelevant for likelihood to be cyber victimized.

\subsection{Gender}

Total 63 out of $87(72.41 \%)$ of male users and 50 out of $66(75.76 \%)$ of female SNS users have never faced cyberbullying. The $(p=)$ value for this analysis is 0.90 which is $(p>0.05)$ greater than the significance level which allows us to accept the null hypothesis for $\mathrm{H} 2$. Also, looking at the numbers $14.94 \%$ and $12.64 \%$ respectively of males and female students having been bullied which are very close to each other. Hence, from the observation and statistical analysis, we can state that gender does not play a substantial role in the cyber victimization of the students as male students are bullied only slightly more compared to female students.

\subsection{Personality}

As mentioned above, we will be using the 15 Likert-Scale questions derived from [59]. Each of the 15 questions acts as a sub-variable for each factor from the FiveFactor model.

A principal component analysis (PCA) was conducted on 15 variables with orthogonal rotation (varimax). The procedure was adopted and followed from [67]. The Kaiser-Meyer-Olkin measure verified the sampling adequacy for the analysis, KMO = .69 which is mediocre but well above the acceptable limit of .5 [68]. Two factors, $E c$ and $C a$, were below the KMO threshold clocking 0.45 and 0.49 respectively. Hence, we had to discard them in the further analysis. Bartlett's test of sphericity, $X^{2}(78)=$ 538.63, $p<.001$, indicated that correlations between items was sufficiently large for PCA. An initial analysis was run to obtain eigenvalues for each component of data. Assuming the number of samples and Kaiser's criterion, we considered five components for final analysis. The items in the cluster on the same components suggest component 1 represents openness to experience, component 2 is neuroticism and component 3 is extroversion followed by components 4 and 5 that are agreeableness and conscientiousness. From the reliability analysis, the variables openness to experience, neuroticism and agreeableness of the personality data have high reliability $(\alpha=0.89,0.71$, and 0.68$)$. However, the variables extroversion and conscientiousness have low reliability ( $\alpha=0.53$ and 0.45 ). As stated by Unwin (2013), still it is not a concern for our data as the threshold for Cronbach's $\alpha=0.3$. From the analysis above, we have gathered information that $18.98 \%$ respondents possess the personality openness to experience, $20.88 \%$ are neurotics, $16.45 \%$ are the extroverts, $20.88 \%$ possess the personality of agreeableness and $22.81 \%$ possess conscientiousness.

The overall percentage of respondents getting cyberbullied is as low as $13.73 \%$ whereas, the people who are unsure and responded maybe is $12.42 \%$. The percentage of respondents being bullied is high with the personality openness to experience. With $17.24 \%$ of the respondents facing cyberbullying and $20.69 \%$ unsure, openness to experience is the personality trait which is slightly more likely to be cyber victimized compared to the other personality traits, whereas, the respondents with the personality trait agreeableness are the least cyberbullied with $82.76 \%$.

\section{Discussion}

We began our research with a framework that included different traits of cyberbullying and its related terminologies. Figure 1 illustrates the conceptual framework around which we designed our research. This framework is a preliminary model that we are proposing to understand the area of this research.

Since the initial phases, we have tried to uncover all the possible areas which concern cyberbullying, and which could have led to the outcome of our research. The area which we specifically tried to uncover in our research are social media users, their characteristics (age, gender, and personality) and how they lead to cyber victimization of an individual. Based on this conceptual framework, we designed and implemented the methodology explained above. The results we found are, "there is no visible 
correlation of cyberbullying with respect to age and gender in New Zealand, whereas, the students with the personality trait openness to experience is slightly more prone than people with other personalities."

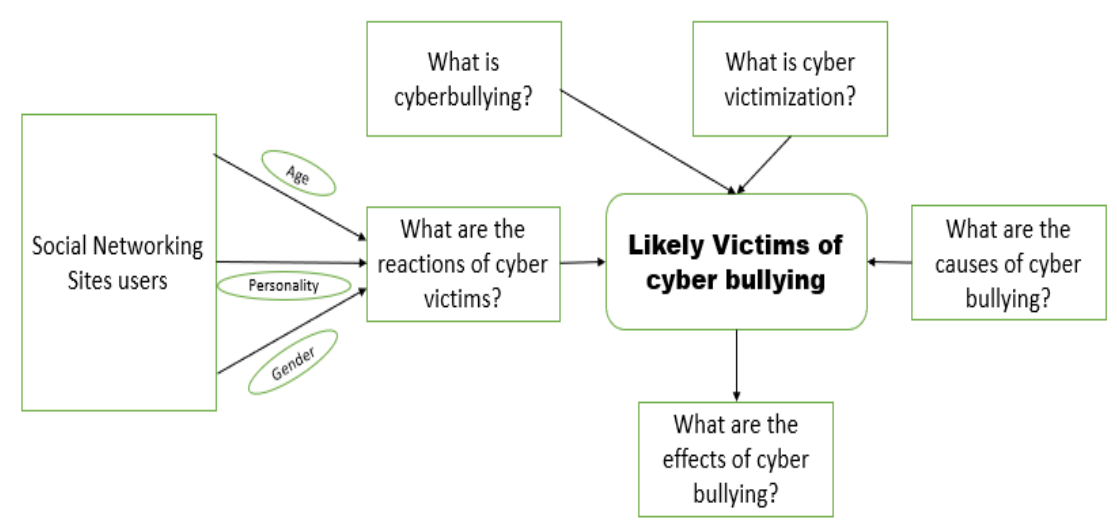

Fig. 1. Cyberbullying Research Framing

When we investigated our first factor of the research i.e. the age of the students, there was absolutely no correlation between the age of the university students and cyber victimization. In the literature, we came across a major difference of opinions among different researchers. [44], in his early studies, stated that an individual using the internet is always prone to cyberbullying irrespective of his age. The same view was supported by [62], who state that cyberbullying gradually decreases in adolescence. We have seen studies which proved the relatability of cyber victimization which exists among youth $[11,14,50-53]$. Based on this we designed a hypothesis which said the age of the student on social media has a direct impact on them being cyber victimised. But our results give us a contradictory result validating the null hypothesis. The results are not as strange as they seem because of similar claims made in past studies [46-49]. One of the reasons for these results may be the nature of sample we have collected. The sample population was university students who are older than school children and have bigger problems i.e. career, finance etc. to deal with. Another reason may be reluctance to admit, or ignorance towards the problem.

In previous literature, there has been a major inconsistency while finding a pattern for cyber victimization with respect to gender difference. Still, some literature has led our research to some other perspectives than based on gender disparity. Studies on traditional bullying state that males possess more bullying behaviour than females [18],[69]. Based on the literature we have seen, we presented a hypothesis "the gender of the student on social media has a direct impact on their being cyber victimised." But our research has presented us with contradictory results. Not only have university students of both genders been cyber victimized equally but the students who have never been cyber victimized are also somewhat similar. This means that gender plays no part in the cyber victimization of university students in New Zealand. There should be some rational explanation for this which can be uncovered by qualitative research and interviewing different people with different genders. This will give us a perspective on why gender does not play a part in cyber victimization in New Zealand.

Few studies have been conducted in this domain with the five-factor personality traits by [59], but those few studies have demonstrated how a different personality can play a part in the cyber victimization of an individual $[30,32,70]$. Though the number of students cyberbullied is not substantial, the slight difference between the factorsbased result and actual results look significant. The detail regarding the percentage of students belonging to the personality trait of openness to experience is higher than the percentage of total cyberbullied. On the other hand, the percentage of students not cyberbullied with the same personality trait is much lower than the actual number. During hypothesis development, we discussed that the students belonging to the personality trait openness to experience are likely to be cyberbullied, as they could be trying to move out of their comfort zone. Increased novelty seeking that is linked to 
openness to experience has been associated with internet addiction [71]. The personality trait openness to experience is associated mainly with fantasy, aesthetics, feelings, actions, ideas, and values [72]. It has also been discussed in the research that drug usage by this personality is higher than others [73]. The research conducted by [74], confirms the target youth who are more prone to addictions like smoking, drinking etc. can also be the victims of cyberbullying. This gives us a fair idea how likely the students with the personality trait openness to experience are prone to be cyberbullied. Moreover, the students in a dilemma with the answer 'maybe' for the personality openness to experience also has the higher percentage compared to the total population. Hence, the personality openness to experience is more susceptible to be cyberbullied as compared to its counterparts.

\section{Conclusion, Limitations and Future work}

The core research topic of this project was to identify the characteristics of the cyber victims on social media in New Zealand. We can demonstrate how age and gender don't play any substantial part in the cyber victimization of students, whereas the personality trait openness to experience may lead to cyberbullying by others to a certain extent. This perspective may play an important part in future research in the field of cyber victimization.

As personality plays part in the cyber bullying of an individual, policies, interventions, and monitoring are some of the measures to be taken. Further research can be carried out to determine the earliest age children have access to social media. Based on that number, a small survey can be carried out in a school to determine the personality of every individual child. The children with the personality trait openness to experience can be monitored to avoid cyber bullying. Here, some implications of [42] data mining technologies and artificial intelligence methods can be applied for avoiding cyberbullying. In section 5.4 we identified Facebook as the most used social media by the students with personality trait openness to experience. This can also help us to monitor the specific social media to evade cyberbullying of these students. Generic internet safety tips and cyberbullying prevention measures can be incorporated at home, in universities, and the social media homepages. In section 2.4 we discussed a recent algorithm proposed by [41]. This algorithm theorises by considering the potential reasons for cyberbullying. It has two factors i.e. Neutralization and Deterrence theories. The neutralization theory aims for the people who are more prone to cyberbullying whereas the deterrence theory acts as its antidote. In such cases, our findings can also contribute as one of the factors. The students possessing the personality trait openness to experience can be aimed in the neutralization theory.

\subsection{Limitations and Future work}

From the results, we can see the number of students who have never been bullied is high within the age group of 31 years and above. This leads to a counter-argument that, the older the person, the less likely they are to be cyber victimized. Because there has been a gradual drop in the number of people being cyber victimized in later years, we can focus on the younger population. There can be future work in this area of research on school children in New Zealand to see whether young adults i.e. from the age 13 to 19 years having access to the technology are more likely to be cyber victimized. For our research, we analysed 158 responses. Amongst these responses, we had a gender imbalance. Most them are male responses. In future work, we could gather more data with a larger time span. If we got enough data, we could use the random 50\% male population and $50 \%$ female for analysis purposes. This would give us an accurate ratio of the cyber victim's gender-wise. This could also provide us with the more substantial evidence on whether the victims of either gender are more likely to be cyberbullied.

After conducting the quantitative data analysis and interpreting the results, we have realised there is an immense scope for qualitative research. The qualitative research would give us an interpretive, naturalistic approach to the subject matter [23]. In the research done so far, for example in the quantitative research if we are asking the question "Have you ever been cyberbullied?" in the qualitative research we can first 
ask the question to a sample population "What does cyberbullying mean to you?" This kind of question in the qualitative research and analysis will give us the implications to the questions to follow in the quantitative research. This could be a good implementation in future work as well. One more important aspect we can monitor is the cultural difference. According to [75], culture is defined as "a collective programming of the mind that distinguishes two different people belonging to two different groups". It is not necessary that two individuals in the same group should be like each other. These can be two completely different individuals who lead their lives in a similar manner [76]. When we look it into the New Zealand perspective, no major study has been found considering this factor. For instance, among the North American communities, Spanish-speaking or Hispanic youth are frequent bullies compared to other ethnicities like African-Americans or Caucasians [69]. Whereas, it is the other way around when it comes to victimization as African-Americans are less bullied compared to the other two ethnicities mentioned above [77],[78]. As per the report published by [79], people in New Zealand can be identified as four major ethnicities: European, Maori, Asian and Pacifica. We tried to implement this factor but unfortunately, the numbers were not on the higher side. Moreover, most of the respondents were either Indians or Europeans. As mentioned above, for the future research if we can gather more numbers, this study can also prove interesting.

In conclusion, the research addresses the gap in the initial phase and has overcome it in later phases. The research gap was in regards with the work done so far in the field cyberbullying in New Zealand. We have implemented the quantitative approach for identifying the characteristics of cyber victims on social media in New Zealand. However, the study also provides us with an insight on how the rate of cyberbullying is low in New Zealand. It has provided a greater clarification surrounding likely leading to the future research outputs.

\section{Appendix}

The survey questions can be requested from the authors.

\section{References}

1. Olweus, D., Cyber Bullying. Aggression and Violence: A Social Psychological Perspective, 2016.

2. Bauman, S., R.B. Toomey, and J.L. Walker, Associations among bullying, cyberbullying, and suicide in high school students. Journal of adolescence, 2013. 36(2): p. 341-350.

3. Cassidy, W., C. Faucher, and M. Jackson, Cyberbullying among youth: A comprehensive review of current international research and its implications and application to policy and practice. School Psychology International, 2013: p. 0143034313479697.

4. Holt, M.K., et al., Multidisciplinary approaches to research on bullying in adolescence. Adolescent Research Review, 2017. 2(1): p. 1-10.

5. Chandrashekhar, A., G. Muktha, and D. Anjana, Cyberstalking and Cyberbullying: Effects and prevention measures. Imperial Journal of Interdisciplinary Research, 2016. 2(3): p. 95102.

6. Perren, S., et al., Bullying in school and cyberspace: Associations with depressive symptoms in Swiss and Australian adolescents. Child and adolescent psychiatry and mental health, 2010. 4(1): p. 28.

7. Price, M. and J. Dalgleish, Cyberbullying: Experiences, impacts and coping strategies as described by Australian young people. Youth Studies Australia, 2010. 29(2): p. 51.

8. Kljakovic, M., C. Hunt, and P. Jose, Incidence of bullying and victimisation among adolescents in New Zealand. New Zealand Journal of Psychology, 2015. 44(2).

9. Hinduja, S. and J.W. Patchin. Cyberbullying statistics. 2014; Available from: http://www.puresight.com/Cyberbullying/cyber-bullying-statistics.html.

10. Bayzick, J., A. Kontostathis, and L. Edwards, Detecting the presence of cyberbullying using computer software. 2011.

11. Maher, D., Cyberbullying: An ethnographic case study of one Australian upper primary school class. Youth Studies Australia, 2008. 27(4): p. 50.

12. Willard, N., Cyberbullying and cyberthreats. Eugene, OR: Center for Safe and Responsible Internet Use, 2006.

13. Willard, N.E., Cyberbullying and cyberthreats: Responding to the challenge of online social aggression, threats, and distress. 2007: Research Press. 
14. Patchin, J.W. and S. Hinduja, Bullies move beyond the schoolyard: A preliminary look at cyberbullying. Youth violence and juvenile justice, 2006. 4(2): p. 148-169.

15. Limber, S.P. and M.A. Small, State laws and policies to address bullying in schools. School Psychology Review, 2003. 32(3): p. 445-456.

16. Campbell, M.A., Cyber bullying: An old problem in a new guise? Journal of Psychologists and Counsellors in Schools, 2005. 15(1): p. 68-76.

17. Craig, W.M., The relationship among bullying, victimization, depression, anxiety, and aggression in elementary school children. Personality and individual differences, 1998. 24(1): p. 123-130.

18. Forero, R., et al., Bullying behaviour and psychosocial health among school students in New South Wales, Australia: cross sectional survey. Bmj, 1999. 319(7206): p. 344-348.

19. Hodges, E.V. and D.G. Perry, Personal and interpersonal antecedents and consequences of victimization by peers. Journal of personality and social psychology, 1999. 76(4): p. 677.

20. Roland, E., Bullying, depressive symptoms and suicidal thoughts. Educational research, 2002. 44(1): p. 55-67.

21. Rates of cyber bullying in New Zealand alarming, in NZ Herald. 2016.

22. Awiria, O., D. Olweus, and B. Byrne, Bullying at School-What We Know and What We Can DoCoping with Bullying in Schools. 1994.

23. Crick, N.R. and J.K. Grotpeter, Relational aggression, gender, and social-psychological adjustment. Child development, 1995. 66(3): p. 710-722.

24. Williams, K.R. and N.G. Guerra, Prevalence and predictors of internet bullying. Journal of adolescent health, 2007. 41(6): p. S14-S21.

25. Jose, P.E., et al., The joint development of traditional bullying and victimization with cyber bullying and victimization in adolescence. Journal of Research on Adolescence, 2012. 22(2): p. 301-309.

26. Ybarra, M.L. and K.J. Mitchell, Youth engaging in online harassment: Associations with caregiver-child relationships, Internet use, and personal characteristics. Journal of adolescence, 2004. 27(3): p. 319-336.

27. Li, Q., Cyberbullying in schools: A research of gender differences. School psychology international, 2006. 27(2): p. 157-170.

28. Slonje, R., P.K. Smith, and A. FriséN, The nature of cyberbullying, and strategies for prevention. Computers in human behavior, 2013. 29(1): p. 26-32.

29. Björkqvist, K., Sex differences in physical, verbal, and indirect aggression: A review of recent research. Sex roles, 1994. 30(3): p. 177-188.

30. Paulhus, D.L. and K.M. Williams, The dark triad of personality: Narcissism, Machiavellianism, and psychopathy. Journal of research in personality, 2002. 36(6): p. 556563.

31. Geis, F. and M. Levy, Studies in Machiavellianism. 1970, Elsevier.

32. Raskin, R.N. and C.S. Hall, A narcissistic personality inventory. Psychological reports, 1979.

33. Reidy, D.E., et al., Effects of narcissistic entitlement and exploitativeness on human physical aggression. Personality and Individual Differences, 2008. 44(4): p. 865-875.

34. Ang, R.P., K.-A. Tan, and A. Talib Mansor, Normative beliefs about aggression as a mediator of narcissistic exploitativeness and cyberbullying. Journal of interpersonal violence, 2011. 26(13): p. 2619-2634.

35. Görzig, A. and K. Ólafsson, What makes a bully a cyberbully? Unravelling the characteristics of cyberbullies across twenty-five European countries. Journal of Children and Media, 2013. 7(1): p. 9-27.

36. Görzig, A., Who bullies and who is bullied online?: a study of 9-16 year old internet users in 25 European countries. 2011.

37. Dooley, J., J. Pyzalski, and D. Cross, Cyberbullying and face-to-face bullying: Similarities and differences. Zeitschrift für Psychologie/Journal of Psychology, 2009. 217(4): p. 182188.

38. Heirman, W. and M. Walrave, Assessing concerns and issues about the mediation of technology in cyberbullying. Cyberpsychology: Journal of Psychosocial Research on Cyberspace, 2008. 2(2).

39. Spears, B., et al., Behind the scenes and screens: Insights into the human dimension of covert and cyberbullying. Zeitschrift für Psychologie/Journal of Psychology, 2009. 217(4): p. 189-196.

40. Smith, P.K., et al., Cyberbullying: Its nature and impact in secondary school pupils. Journal of child psychology and psychiatry, 2008. 49(4): p. 376-385.

41. Zhang, S., et al., Friend or Foe: Cyberbullying in Social Network Sites. ACM SIGMIS Database, 2016. 47(1): p. 51-71.

42. Zhao, R., A. Zhou, and K. Mao. Automatic detection of cyberbullying on social networks based on bullying features. in Proceedings of the 17th International Conference on Distributed Computing and Networking. 2016. ACM.

43. Xu, J.-M., et al. Learning from bullying traces in social media. in Proceedings of the 2012 
conference of the North American chapter of the association for computational linguistics: Human language technologies. 2012. Association for Computational Linguistics.

44. Witmer, D.F., Risky Business: Why People Feel Safe in Sexually Expicit On-Line Communication. Journal of Computer-Mediated Communication, 1997. 2(4): p. 0-0.

45. Slonje, R. and P.K. Smith, Cyberbullying: Another main type of bullying? Scandinavian journal of psychology, 2008. 49(2): p. 147-154.

46. Beran, T. and Q. Li, The relationship between cyberbullying and school bullying. The Journal of Student Wellbeing, 2008. 1(2): p. 16-33.

47. Didden, R., et al., Cyberbullying among students with intellectual and developmental disability in special education settings. Developmental neurorehabilitation, 2009. 12(3): p. 146-151.

48. Katzer, C., D. Fetchenhauer, and F. Belschak, Cyberbullying: Who are the victims? A comparison of victimization in Internet chatrooms and victimization in school. Journal of Media Psychology, 2009. 21(1): p. 25-36.

49. Varjas, K., J. Meyers, and M. Hunt, Student survey of bullying behavior-revised 2 (SSBBR2). Atlanta, GA: Georgia State University, Center for Research on School Safety, School Climate and Classroom Management, 2006.

50. DeHue, F., C. Bolman, and T. Völlink, Cyberbullying: Youngsters' experiences and parental perception. CyberPsychology \& Behavior, 2008. 11(2): p. 217-223.

51. Kowalski, R.M. and S.P. Limber, Electronic bullying among middle school students. Journal of adolescent health, 2007. 41(6): p. S22-S30.

52. Ybarra, M.L., M. Diener-West, and P.J. Leaf, Examining the overlap in Internet harassment and school bullying: Implications for school intervention. Journal of Adolescent Health, 2007. 41(6): p. S42-S50.

53. Ybarra, M.L. and K.J. Mitchell, How risky are social networking sites? A comparison of places online where youth sexual solicitation and harassment occurs. Pediatrics, 2008. 121(2): p. e350-e357.

54. Erdur-Baker, Ö., Cyberbullying and its correlation to traditional bullying, gender and frequent and risky usage of internet-mediated communication tools. New media \& society, 2010. 12(1): p. 109-125.

55. Boulton, M.J. and K. Underwood, Bully/victim problems among middle school children. British Journal of Educational Psychology, 1992. 62(1): p. 73-87.

56. Lagerspetz, K.M., et al., Group aggression among school children in three schools. Scandinavian Journal of Psychology, 1982. 23(1): p. 45-52.

57. O'moore, A. and B. Hillery, Bullying in Dublin schools. The Irish Journal of Psychology, 1989. 10(3): p. 426-441.

58. Stephenson, P. and D. Smith, Bullying in the junior school. Bullying in schools, 1989: p. 45-57.

59. McCrae, R.R. and P.T. Costa Jr, Personality trait structure as a human universal. American psychologist, 1997. 52(5): p. 509.

60. Utz, S., Show me your friends and I will tell you what type of person you are: How one's profile, number of friends, and type of friends influence impression formation on social network sites. Journal of Computer-Mediated Communication, 2010. 15(2): p. 314-335.

61. Amichai-Hamburger, Y. and G. Vinitzky, Social network use and personality. Computers in human behavior, 2010. 26(6): p. 1289-1295.

62. Vazsonyi, A.T., et al., Cyberbullying in context: Direct and indirect effects by low selfcontrol across 25 European countries. European Journal of Developmental Psychology, 2012. 9(2): p. 210-227.

63. Iarossi, G., The power of survey design: A user's guide for managing surveys, interpreting results, and influencing respondents. 2006: World Bank Publications.

64. Peluchette, J.V., et al., Cyberbullying victimization: Do victims' personality and risky social network behaviors contribute to the problem? Computers in Human Behavior, 2015. 52: p. 424-435.

65. Vodanovich, S., Digital Native Well-being and Development in Ubiquitous Spaces. 2014, ResearchSpace@ Auckland.

66. Dehne, M. and J. Schupp, Persönlichkeitsmerkmale im Sozio-oekonomischen Panel (SOEP)-Konzept, Umsetzung und empirische Eigenschaften. Research Notes, 2007. 26: p. $1-70$.

67. Unwin, A., Discovering Statistics Using $R$ by Andy Field, Jeremy Miles, Zoë Field. International Statistical Review, 2013. 81(1): p. 169-170.

68. Kaiser, H.F., An index of factorial simplicity. Psychometrika, 1974. 39(1): p. 31-36.

69. Haynie, D.L., et al., Bullies, victims, and bully/victims: Distinct groups of at-risk youth. The Journal of Early Adolescence, 2001. 21(1): p. 29-49.

70. Christie, R. and F.L. Geis, Studies in machiavellianism. 2013: Academic Press.

71. Ko, C.-H., et al., The characteristics of decision making, potential to take risks, and personality of college students with Internet addiction. Psychiatry research, 2010. 175(1): p. 121-125. 
72. Kuss, D.J., M.D. Griffiths, and J.F. Binder, Internet addiction in students: Prevalence and risk factors. Computers in Human Behavior, 2013. 29(3): p. 959-966.

73. Terracciano, A., et al., Five-Factor Model personality profiles of drug users. BMC psychiatry, 2008. 8(1): p. 22.

74. Chan, S.F. and A.M. La Greca. Cyber Victimization and Aggression: Are They Linked with Adolescent Smoking and Drinking? in Child \& Youth Care Forum. 2016. Springer.

75. Hofstede, G., Dimensionalizing cultures: The Hofstede model in context. Online readings in psychology and culture, 2011. 2(1): p. 8.

76. Hofstede, G., Culture's consequences: Comparing values, behaviors, institutions, and organizations across cultures. 2001, Thousand Oaks, CA: Sage.

77. Nansel, T.R., et al., Bullying behaviors among US youth: Prevalence and association with psychosocial adjustment. Jama, 2001. 285(16): p. 2094-2100.

78. Spriggs, A.L., et al., Adolescent bullying involvement and perceived family, peer and school relations: Commonalities and differences across race/ethnicity. Journal of Adolescent Health, 2007. 41(3): p. 283-293.

79. Kenny, K. and A. Fayers. How the ethnic mix in your community is set to change. 201530 September 2015; Available from: http://www.stuff.co.nz/national/72557057/How-theethnic-mix-in-your-community-is-set-to-change. 